\title{
Effect of nitrogen-rich cell culture surfaces on type $X$ collagen expression by bovine growth plate chondrocytes
}

Alain Petit ${ }^{1,2+}$, Caroline N Demers ${ }^{1+}{ }^{1}$ Pierre-Luc Girard-Lauriault ${ }^{3}$, Dorothy Stachura ${ }^{1}$, Michael R Wertheimer ${ }^{3}$, John Antoniou ${ }^{1,2+}$, Fackson Mwale ${ }^{1,2^{*}+}$

* Correspondence: fmwale@ldi.jgh. mcgill.ca

${ }^{1}$ Lady Davis Institute for Medical Research, SMBD-Jewish General Hospital, 3755 Chemin de la Cote Ste-Catherine, Montreal, QC H3T 1E2, Canada

\begin{abstract}
Background: Recent evidence indicates that osteoarthritis (OA) may be a systemic disease since mesenchymal stem cells (MSCs) from OA patients express type $X$ collagen, a marker of late stage chondrocyte hypertrophy (associated with endochondral ossification). We recently showed that the expression of type $X$ collagen was suppressed when MSCs from OA patients were cultured on nitrogen (N)-rich plasma polymer layers, which we call "PPE:N" (N-doped plasma-polymerized ethylene, containing up to 36 atomic percentage (at.\%) of $\mathrm{N}$.
\end{abstract}

Methods: In the present study, we examined the expression of type X collagen in fetal bovine growth plate chondrocytes (containing hypertrophic chondrocytes) cultured on PPE:N. We also studied the effect of PPE:N on the expression of matrix molecules such as type II collagen and aggrecan, as well as on proteases (matrix metalloproteinase-13 (MMP-13) and molecules implicated in cell division (cyclin B2). Two other culture surfaces, "hydrophilic" polystyrene (PS, regular culture dishes) and nitrogen-containing cation polystyrene (Primaria ${ }^{\circledR}$ ), were also investigated for comparison.

Results: Results showed that type X collagen mRNA levels were suppressed when cultured for 4 days on PPE:N, suggesting that type $X$ collagen is regulated similarly in hypertrophic chondrocytes and in human MSCs from OA patients. However, the levels of type $X$ collagen mRNA almost returned to control value after 20 days in culture on these surfaces. Culture on the various surfaces had no significant effects on type II collagen, aggrecan, MMP-13, and cyclin B2 mRNA levels.

Conclusion: Hypertrophy is diminished by culturing growth plate chondrocytes on nitrogen-rich surfaces, a mechanism that is beneficial for MSC chondrogenesis.

Furthermore, one major advantage of such "intelligent surfaces" over recombinant growth factors for tissue engineering and cartilage repair is potentially large cost-saving.

\section{Background}

Endochondral ossification involves the expression of type $\mathrm{X}$ collagen, a marker of chondrocyte hypertrophy [1-3]. Recent evidence indicates that a major drawback of current cartilage- and disc-tissue engineering is that human mesenchymal stem cells (MSCs) from osteoarthritic (OA) patients express type X collagen [4]. We have shown that synthetic polymer surfaces created by glow discharge plasma can suppress the expression of genes associated with hypertrophy in committed human MSCs from OA 
patients [5-7]. However, little is known about the effect of different culture surfaces on gene expression in the case of growth plate chondrocytes.

Endochondral ossification begins during long bone formation in the embryo [8]. After birth, until adulthood, growth of the long bone is centred in the cartilagenous growth plates, leading to an increase in bone length and epiphyseal growth. It is also an essential component of fracture repair. The primary mammalian growth plate can be divided into several zones, namely the resting, proliferative, and hypertrophic zones [8]. The resting zone chondrocytes elaborate an extracellular matrix similar to the proliferating zone cells, one which expresses type II collagen and the proteoglycan aggrecan; these constitute, together with other matrix molecules, an extensive extracellular matrix. In the proliferative zone, chondrocytes divide actively and synthesize different collagen molecules (types II, IX, and XI) and cartilage-specific proteoglycans [1,2,8]. At this point in time, they express cell cycle-related genes such as cyclins [8]. After cessation of cell division, chondrocytes partly resorb their extracellular matrix and enlarge (become hypertrophic) as they express type $\mathrm{X}$ collagen. The up-regulation of type $\mathrm{X}$ collagen expression signals the change in chondrocytic phenotype from prehypertrophic to hypertrophic, after which the matrix of the longitudinal septa between the cells starts to mineralize $[2,8]$. This coordinated proliferation and differentiation of growth plate chondrocytes is required for normal growth and development of the skeleton [9-14].

We recently showed that a novel atmospheric-pressure plasma-polymerized thin film material, named "nitrogen-rich plasma-polymerized ethylene" (PPE:N), is able to inhibit hypertrophy as well as osteogenesis in committed human MSCs from OA patients [6]. In contrast, neither aggrecan nor type I collagen expression were significantly affected. These results indicated that PPE:N coatings may be suitable surfaces for inducing MSCs to a chondrocyte or disc-like (nucleus pulposus) phenotype for tissue engineering of cartilage or intervertebral discs, respectively, in which hypertrophy and osteogenesis must be avoided.

In this study, the effect of culturing growth plate chondrocytes expressing the hypertrophic phenotype (cells that express type X collagen) on PPE:N, Primaria ${ }^{\circledR}$, or regular polystyrene (PS) culture dishes was investigated using reverse transcriptase (RT) and polymerase chain reaction (PCR). Primaria ${ }^{\circledR}$ was chosen because it has been described as having nitrogen-containing cations at its surface $[15,16]$. Thus, we set out to test the hypothesis that the chemically-bound nitrogen content, $[\mathrm{N}]$, may be an important regulator of cellular hypertrophy. We demonstrated that, similarly to what we observed in human MSCs, fetal bovine growth plate hypertrophic chondrocytes respond to $\mathrm{N}$-rich substrates by down-regulation of type $\mathrm{X}$ collagen expression. This may be important in designing substrates for cartilage- or intervertebral disc repair, where prevention of hypertrophy and endochondral ossification is important. Our findings reveal hitherto unsuspected similarities in regulation of expression of type X collagen in these cell types, and they may provide novel insights into how these cells interact with PPE: $\mathrm{N}$ surfaces.

\section{Methods}

\section{A. Deposition of PPE:N}

The methods employed have been described earlier by Girard-Lauriault et al. $[17,18]$. for the experiments reported here, PPE:N films were deposited on biaxially oriented 
polypropylene (BOPP; 3M Company) [6,17-20]. Using this method, films containing 30 at.\% nitrogen, $[\mathrm{N}]$, were deposited from the precursor gas mixture composed of nitrogen $\left(\mathrm{N}_{2}, 10\right.$ standard liters per minute, slm) and ethylene $\left(\mathrm{C}_{2} \mathrm{H}_{4}, 10\right.$ standard cubic centimeters per minute, sccm), the only mixture used in this particular study, unlike our above-referenced earlier work $[6,17,18]$.

To visualize the role of nitrogen content on the attachment of chondrocytes to PPE:N surfaces, micropatterning experiments were carried out as follows: Special $25 \mu \mathrm{m}$ thick Kapton ${ }^{\circledR}$ polyimide masks were placed over the BOPP substrate. These masks were fabricated with an excimer laser, coupled to a precise positioning system, to create square arrays of holes (diameter: $100 \mu \mathrm{m}$; pitch: $200 \mu \mathrm{m}$ ) on an area of $4 \mathrm{~cm}^{2}$. Care was taken to assure intimate contact between the mask and substrate surface during deposition runs.

\section{B. Surface characterization}

The surface compositions of the different cell culture surfaces (PPE:N as well as polystyrene (PS) and Primaria ${ }^{\circledR}$; BD Biosciences, Mississauga, ON) were determined by X-ray photoelectron spectroscopy (XPS) [6,17-20]. Throughout this article, we will be referring to their surface elemental concentrations, $[\mathrm{X}]$, in terms of the elements that comprise them, namely N, C and O; since hydrogen cannot be detected by XPS, $[\mathrm{X}]$ is given by:

$$
[X]=\frac{X}{N+C+O} \times 100 \%
$$

$\mathrm{N}, \mathrm{O}$, and $\mathrm{C}$ being determined from the XPS broad-scan spectra.

\section{Growth plate chondrocyte isolation}

The physes of multiple primary growth plates were isolated from bovine fetuses, as described earlier [1]. These slices ( 2 mm thick) were predominantly from hypertrophic zones. Fetal age, on average 171 days (range 154 - 216 days), was determined by measurement of tibial length [21]. Slices were held for up to $2 \mathrm{~h}$ at room temperature in Dulbecco's modified Eagle's medium (DMEM), pH 7.4, containing (per ml) $100 \mathrm{U}$ penicillin, 100 $\mu \mathrm{g}$ streptomycin (medium A) supplemented with $0.25 \mu \mathrm{g}$ amphotericin $\mathrm{B}$, prior to chondrocyte isolation. The growth plates were digested for 12 to $16 \mathrm{~h}$ using collagenase $(0.7 \%$ $\mathrm{w} / \mathrm{v})$ and hyaluronidase $(0.2 \% \mathrm{w} / \mathrm{v})$ in the presence of medium A, supplemented with $10 \%$ fetal bovine serum (FBS; Hyclone, Logan, UT), as described previously [1].

\section{Cell culture}

After isolation, these heterogeneous chondrocytes expressed type X collagen, a definitive marker of the hypertrophic phenotype (see Results section). Cells were counted with a hemacytometer and 1 million cells in $40 \mu \mathrm{l}$ of medium A, supplemented with $5 \mu \mathrm{g} / \mathrm{ml}$ insulin, $5 \mu \mathrm{g} / \mathrm{ml}$ transferrin, $5 \mathrm{ng} / \mathrm{ml}$ sodium selenite, $1 \mathrm{mg} / \mathrm{ml}$ bovine serum albumin, $60 \mu \mathrm{g} / \mathrm{ml}$ ß-glycerophosphate, and freshly prepared $50 \mu \mathrm{g} / \mathrm{ml}$ ascorbic acid, were carefully pipetted onto the centers of the PPE:N surfaces (covering the entire surface of the wells), Primaria ${ }^{\circledR} 24$-well culture dishes (BD Biosciences), or regular 24-well PS culture dishes. The cells were left to adhere to the surfaces for $1 \mathrm{~h}$. Medium was carefully removed and $2 \mathrm{ml}$ of fresh medium was added. Bovine chondrocytes adhered and grew on the three surfaces. Media was changed every 2 days, up to 20 days in culture. 


\section{E. Total RNA isolation}

Total RNA was extracted from chondrocytes by a modification of the method of Chomcynski and Sacchi [22] using TRIzol reagent (Invitrogen, Burlington, ON). After centrifugation for $15 \mathrm{~min}$ at $12,000 \times g$ at $4^{\circ} \mathrm{C}$, the aqueous phase was precipitated in 0.5 volume of isopropanol, incubated for $10 \mathrm{~min}$ at room temperature, and centrifuged again for $10 \mathrm{~min}$ at $12,000 \times g$ at $4^{\circ} \mathrm{C}$. The resulting RNA pellet was washed in $75 \%$ ethanol, centrifuged for $5 \mathrm{~min}$ at $7,500 \times \mathrm{g}$ at $4^{\circ} \mathrm{C}$, air-dried, resuspended in $25 \mu \mathrm{l}$ of diethylpyrocarbonate-treated water, and assayed for RNA concentration and purity by measuring $A_{260} / A_{280}$.

\section{F. Reverse transcriptase (RT) and polymerase chain reaction (PCR)}

The RT reaction was performed using $0.2 \mu \mathrm{g}$ total RNA isolated from the chondrocytes in a total volume of $20 \mu 1$, containing $50 \mathrm{mM}$ Tris- $\mathrm{HCl}(\mathrm{pH} 8.3), 75 \mathrm{mM} \mathrm{KCl}, 3 \mathrm{mM}$ $\mathrm{MgCl}_{2}, 10 \mathrm{mM}$ DTT, $0.5 \mathrm{mM}$ each dATP, dGTP, dCTP and dTTP, and 200 units of Superscript II ${ }^{\mathrm{TM}}$ RNAse $\mathrm{H}^{-}$reverse transcriptase (Invitrogen).

PCR was performed in a total volume of $25 \mu 1$ containing: $20 \mathrm{mM}$ Tris- $\mathrm{HCl}(\mathrm{pH} 8.4)$, $50 \mathrm{mM} \mathrm{KCl}, 1.5 \mathrm{mM} \mathrm{MgCl}_{2}, 0.2 \mathrm{mM}$ of dATP, dGTP, dCTP, dTTP, $0.8 \mu \mathrm{M}$ of each primer, $1 \mu \mathrm{l}$ of RT mixture and 1.25 units of Taq DNA polymerase (Invitrogen). The 30 cycles of PCR included denaturation $\left(95^{\circ} \mathrm{C}, 1 \mathrm{~min}\right)$, annealing $\left(50^{\circ} \mathrm{C}, 60 \mathrm{sec}\right)$ and extension $\left(72^{\circ} \mathrm{C}, 5 \mathrm{~min}\right)$, as previously described [5-7]. After agarose (2\%) gel electrophoresis, PCR products were visualized by ethidium bromide staining and analyzed using a BioRad VersaDoc image analysis system, equipped with a cooled 12-bit CCD camera (BioRad, Mississauga ON). The intensity of the bands was quantified using Quantity One software on the VersaDoc. 18S rRNA level was used as housekeeping gene and served to normalized the results. The primer sequences used for PCR, shown in Table 1, were chosen because they are specific for bovine mRNA and they amplify a single product.

\section{G. Statistical Analysis}

The non-parametric Kruskal-Wallis test was performed to test for differences between surfaces for each gene, and for changes between the different days of culture. Results were considered significant for $\mathrm{p}<0.05$.

\section{Results}

\section{A. Characteristics of polystyrene, Primaria ${ }^{\circledR}$, and PPE:N coatings}

X-ray photoelectron spectroscopy (XPS) broad-scan spectra of the different surfaces show peaks characteristic of $\mathrm{N}, \mathrm{O}$, and $\mathrm{C}$ (Figure 1). The concentrations of elements are expressed as atomic (at.) percentages (\%); those of $\mathrm{N}$ were $0 \%, 6 \%$, and $29.5 \%$ (all at. \%) for PS, Primaria ${ }^{\circledR}$, and PPE:N, respectively (Table 2). The respective

Table 1 Primer sequences used for PCR

\begin{tabular}{lrc}
\hline Genes & Primers & $\begin{array}{l}\text { Size } \\
(\mathbf{b p})\end{array}$ \\
\hline Aggrecan & 5-CAGAACATGCGCTCCAATGA-3' 5-CGTCATAGGTTCGTTGGTG-3' & 371 \\
Type II collagen & 5-AACCCAGAAACAACACAATCC-3' 5'-GAGGGGAGAAAAGTCCGAAC-3' & 168 \\
Type X collagen & 5'-CTGAGCGATACCAAACACC-3' 5'-CCTCTCAGTGATACACCTTAC-3' & 128 \\
MMP-13 Cyclin B2 & 5'-GATAAAGACTATCCGAGAC-3' 5'-CGAACAATACGGTACTC-3' 5'- & 168 \\
& GTTGACTATGACATGGTG-3' 5'-CAAGACAAAGTGCACGAAC-3' & 358 \\
18S rRNA & 5'-CTACTTGGATAACTGTGGTAATTC-3' 5'-GACTCTAGATAACCTCG-3' & 168 \\
\hline
\end{tabular}




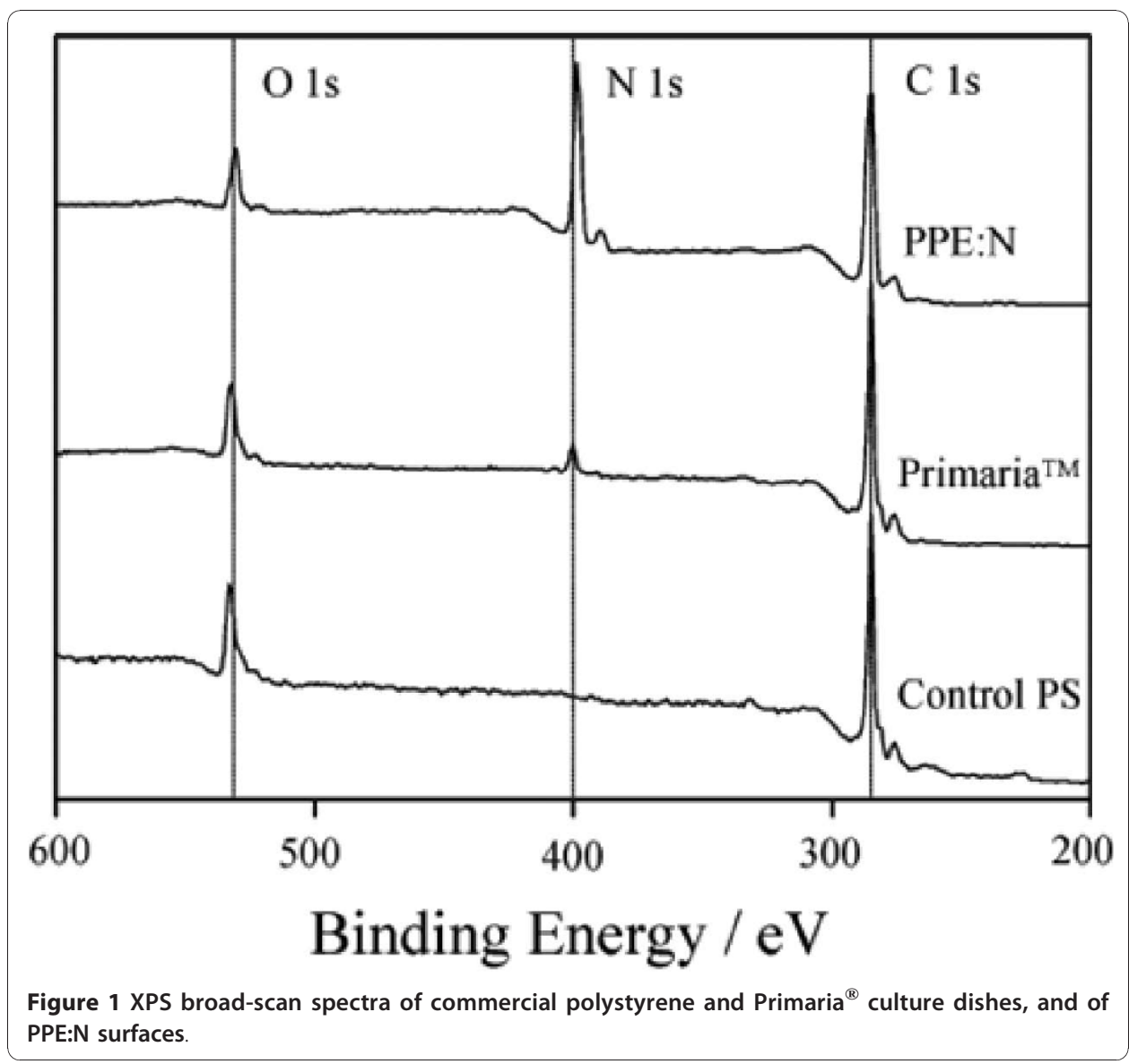

concentration of $\mathrm{O}$ were $18 \%, 15 \%$, and $5 \%$, while those of $\mathrm{C}$ were $82 \%, 79 \%$, and $65.5 \%$ for PS, Primaria ${ }^{\circledR}$, and PPE:N, respectively, again all in at.\%.

\section{B. Micropatterning}

Figure 2 shows that chondrocytes cultured on micro-patterned surfaces preferentially grow on nitrogen-rich surfaces (Figure 2A), indicating that the nitrogen-containing functional groups (notably primary amines, see further below) may be responsible for inducing the attachment of chondrocytes. However, the surface of cell attachment exceeded the mask hole $(100 \mu \mathrm{m})$ in some places, indicating that chondrocytes also grew on the BOPP substrate, but to a lesser extent, or that contact between the mask and substrate surface was not perfectly intimate and that some functional nitrogen groups were formed around the hole. Results also showed that cells that adhere to nitrogen surfaces expressed proteoglycans, as visualized by Safranin-O staining (Figure 2B).

Table 2 Elemental compositions from XPS analyses of the polystyrene, Primaria ${ }^{\circledR}$, and PPE:N surfaces

\begin{tabular}{lccc}
\hline Culture Surfaces & N (at. \%) & O (at. \%) & C (at. \%) \\
\hline Control polystyrene & 0 & 18 & 82 \\
Primaria $^{\circledR}$ & 6 & 15 & 79 \\
PPE:N & 29.5 & 5 & 65.5
\end{tabular}

Note: Hydrogen cannot be detected by XPS. 

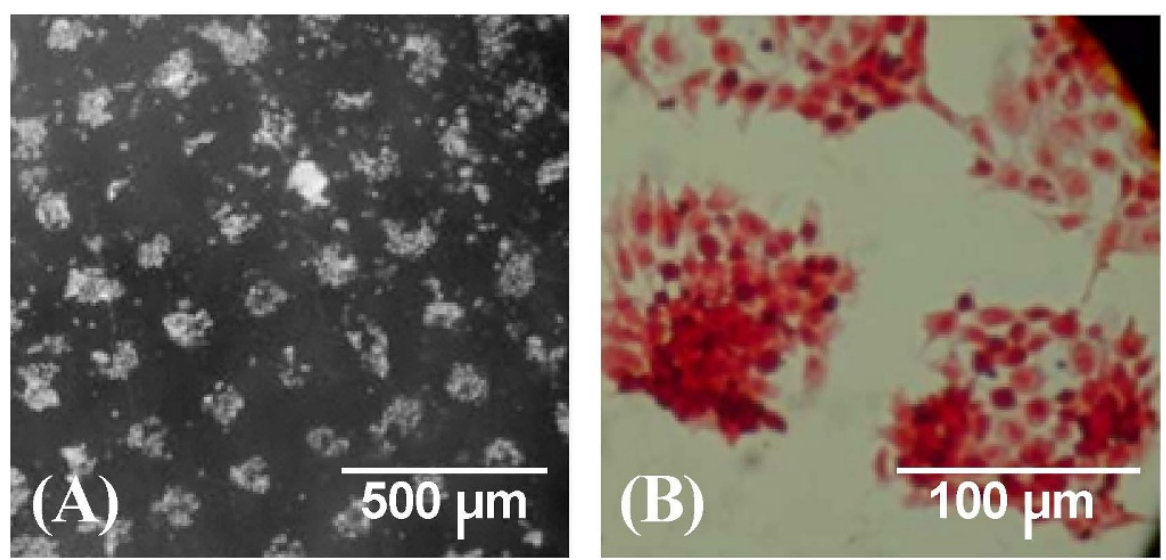

Figure 2 Micro-patterning of growth plate chondrocytes on a PPE:N-coated surface. Kapton ${ }^{\circledR}$ polyimide masks (100 $\mu \mathrm{m}$ openings, $200 \mu \mathrm{m}$ pitch) were placed over the BOPP substrate to create the pattern. Growth plate chondrocytes grown on this patterned surface for 9 days and photomicrographs were taken (A). Proteoglycan production was visualized using Safranin-O staining (B).

\section{Gene expression}

We recently showed that PPE:N surfaces decreased the expression of type $\mathrm{X}$ collagen in MSCs from OA patients. Here, we explored the effect of PPE:N surfaces on the expression of this gene in bovine growth plate chondrocytes. Bovine chondrocytes adhered to the three surfaces compared in the present study and covered the entire surface of the dishes by the end of the culture period (20 days) (results not shown). Figure 3 demonstrates that fetal bovine growth plate chondrocytes isolated without separating the different subpopulations, were enriched with terminally differentiated cells that are characterized by the expression of type X collagen (Day 0), a marker of hypertrophic chondrocytes.

The expression of type $\mathrm{X}$ collagen mRNA was significantly lower after 4 days of culture $(\mathrm{p}=0.007)$ when chondrocytes were cultured on PPE:N, compared to control PS and Primaria ${ }^{\circledR}$, suggesting that type $\mathrm{X}$ collagen is regulated similarly in hypertrophic chondrocytes and in human MSCs from OA patients (Figure 3). However, and contrary to what was observed in MSCs [5-7], the level of type X collagen mRNA almost returned to control value after 20 days in culture on PPE:N surfaces.

Since growth plate chondrocytes secrete an extensive hyaline extracellular matrix consisting principally of type II collagen and the large aggregating proteoglycan aggrecan, we decided to examine the effect of the different culture surfaces on the expression of these genes in growth plate chondrocytes. The levels of type II collagen mRNA did not vary significantly in cells cultured for 4 or 20 days on PS ( $>0.05$ ) (Figure 4): In contrast to type $\mathrm{X}$ collagen, type II collagen mRNA levels were not significantly affected by PPE:N surfaces. The different surfaces and times in culture had little effect on levels of aggrecan mRNA (Figure 5).

Collagenase 3 (MMP-13) was examined because it is the most important collagenase found in the growth plate [23], although MMP-16 is also present. We have shown that MMP-13 is up-regulated during chondrocyte hypertrophy in the growth plate [1,2]. Therefore, we next compared the expression of MMP-13 in growth plate chondrocytes cultured on the different surfaces (Figure 6). MMP-13 mRNA levels were expressed 


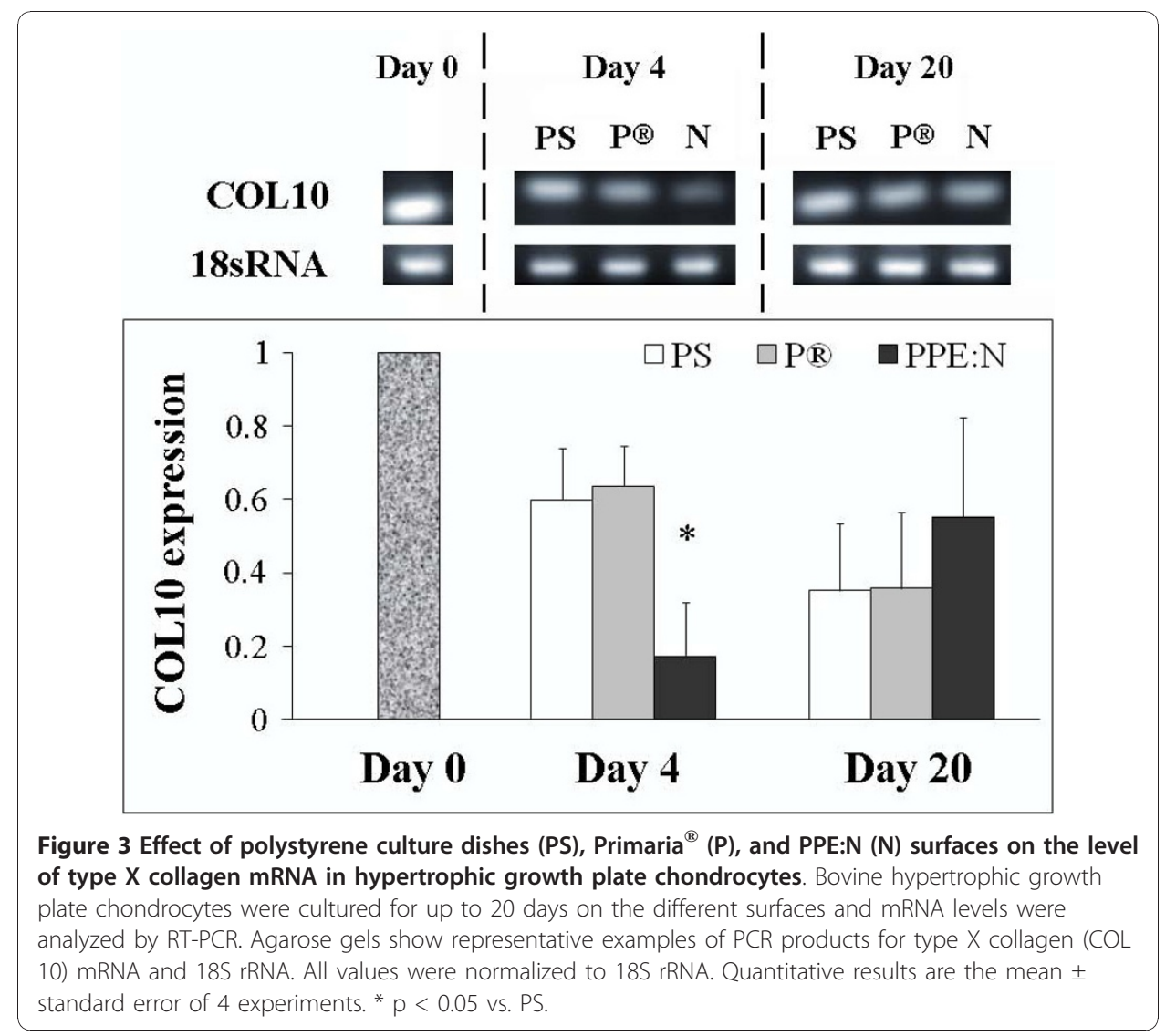

maximally on day 4 and declined significantly on day $20(\mathrm{p}=0.04)$. However, there were no noteworthy differences in its levels in cells cultured on the three different surfaces.

Since the upregulation of MMP-13 expression is observed immediately before and at the onset of cell division, as defined by cyclin B2 expression, and again in chondrocytes that undergo hypertrophy [3], we next analyzed the effect of the different culture surfaces on cyclin B2 mRNA levels (Figure 7). Cyclin B2 mRNA was also expressed maximally on day 4 and declined significantly on day 20 . Here too, there were no appreciable differences in its levels in growth plate chondrocytes cells cultured on the three different surfaces.

\section{Discussion}

The role of cell-biomaterial interactions in tissue engineering is still quite poorly understood. It has been known for some time that cells may be sensitive to subtle differences in surface chemistry [5-7,15,16,24-31]. The chemical and topographical nature of the surface can directly influence cellular responses [5,15,16,18,25,29-32], ultimately affecting the rate and quality of new tissue formation $[25,33,34]$. Our previous studies have shown that PPE:N suppresses the expression not only of type X collagen in MSCs from OA patients, but also of osteogenic marker genes such as alkaline phosphatase, bone sialoprotein, and osteocalcin [6]. In contrast, neither aggrecan nor type I collagen expression were found to be significantly affected. Furthermore, plasma-modified polypropylene or nylon- 6 was found to affect type X collagen expression in human MSCs from OA patients [5,7]. 


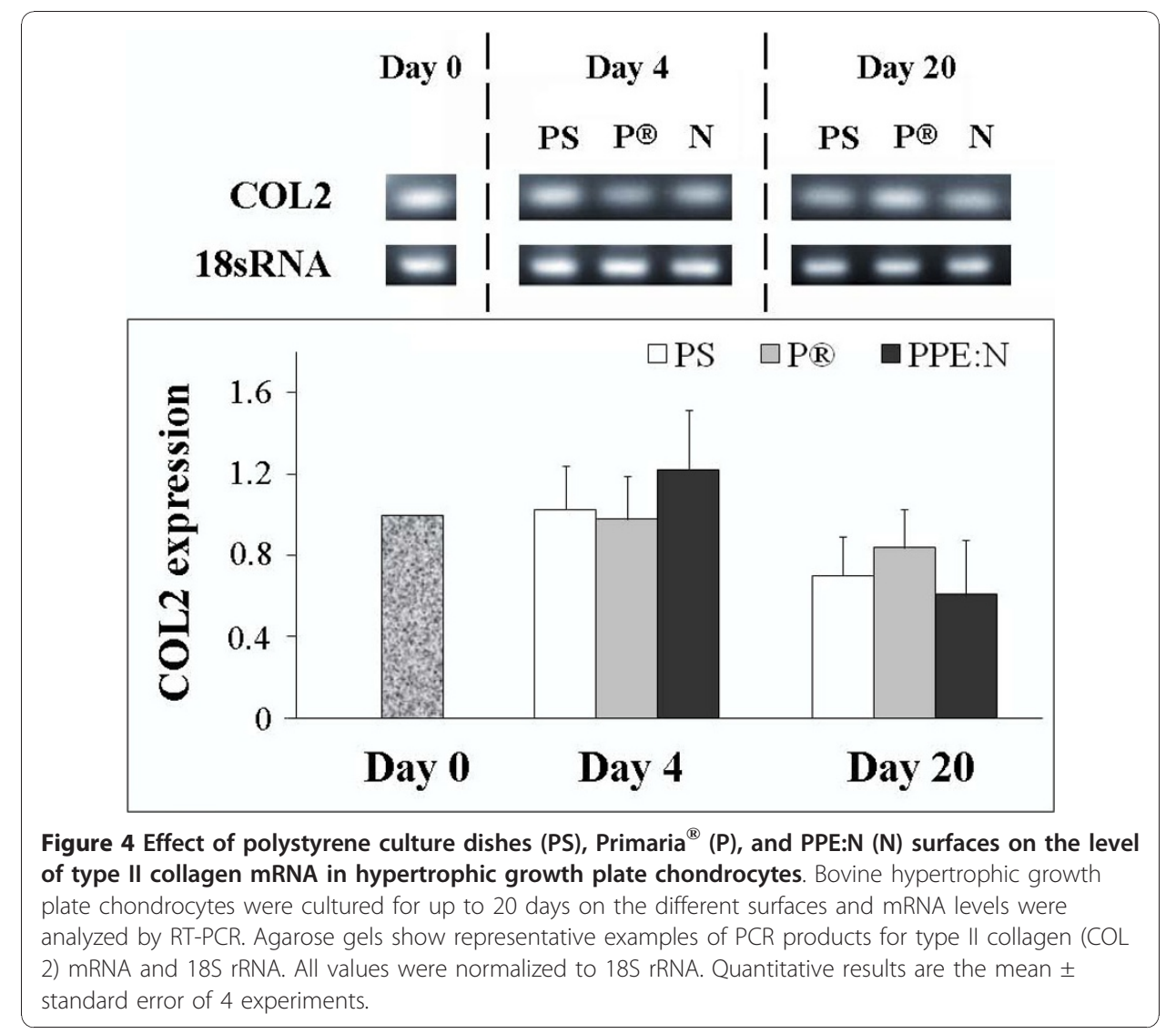

Recent studies have attempted to use growth factors to inhibit type $\mathrm{X}$ collagen expression [35]. However, no earlier study had so far addressed the possible effect of the substratum on growth plate chondrocyte hypertrophy. The recent advances in our group in creating novel bioactive synthetic polymer surfaces with the aid of ultravioletphotochemical and plasma-chemical processes [5,17-19,29], particularly the latter, have enabled us to study the culture of MSCs on nitrogen-enriched (nitrogen concentration, $[\mathrm{N}]$, up to $\sim 20 \%$ ) surface-modified polymers such as BOPP and Nylon-6 polyamide $[5,7]$, as well as on super-rich $([\mathrm{N}] \geq 25 \%)$ plasma-polymerized thin films, PPE:N $[6,17,18]$. The latter substrates have distinguished themselves by their ability to adhere certain cell types that earlier resisted adhesion to all prior-known cell culture surfaces, for example, human U937 monocytes [17,18,29].

The present data indicate that surfaces with high $[\mathrm{N}]$ values are capable of suppressing type $\mathrm{X}$ collagen mRNA in growth plate chondrocytes, with no significant effects on type II collagen, aggrecan, MMP-13 and cyclin B2 mRNA levels. The similarity in type X collagen suppression by PPE:N in hypertrophic growth plate chondrocytes and in human MSCs from OA patients raises the question of whether similar mechanisms are involved. However, contrary to what was observed in MSCs in which PPE:N reduced type $X$ collagen for long time in culture [6], PPE:N was found to decrease the expression of type $\mathrm{X}$ collagen in growth plate chondrocytes only in the short-term. In fact, the decreased type X collagen expression was not observed after 12 days of culture (results not shown). This suggests that the decrease may be associated with the initial preferential adhesion of growth plate chondrocytes, as illustrated in Figure 2. 


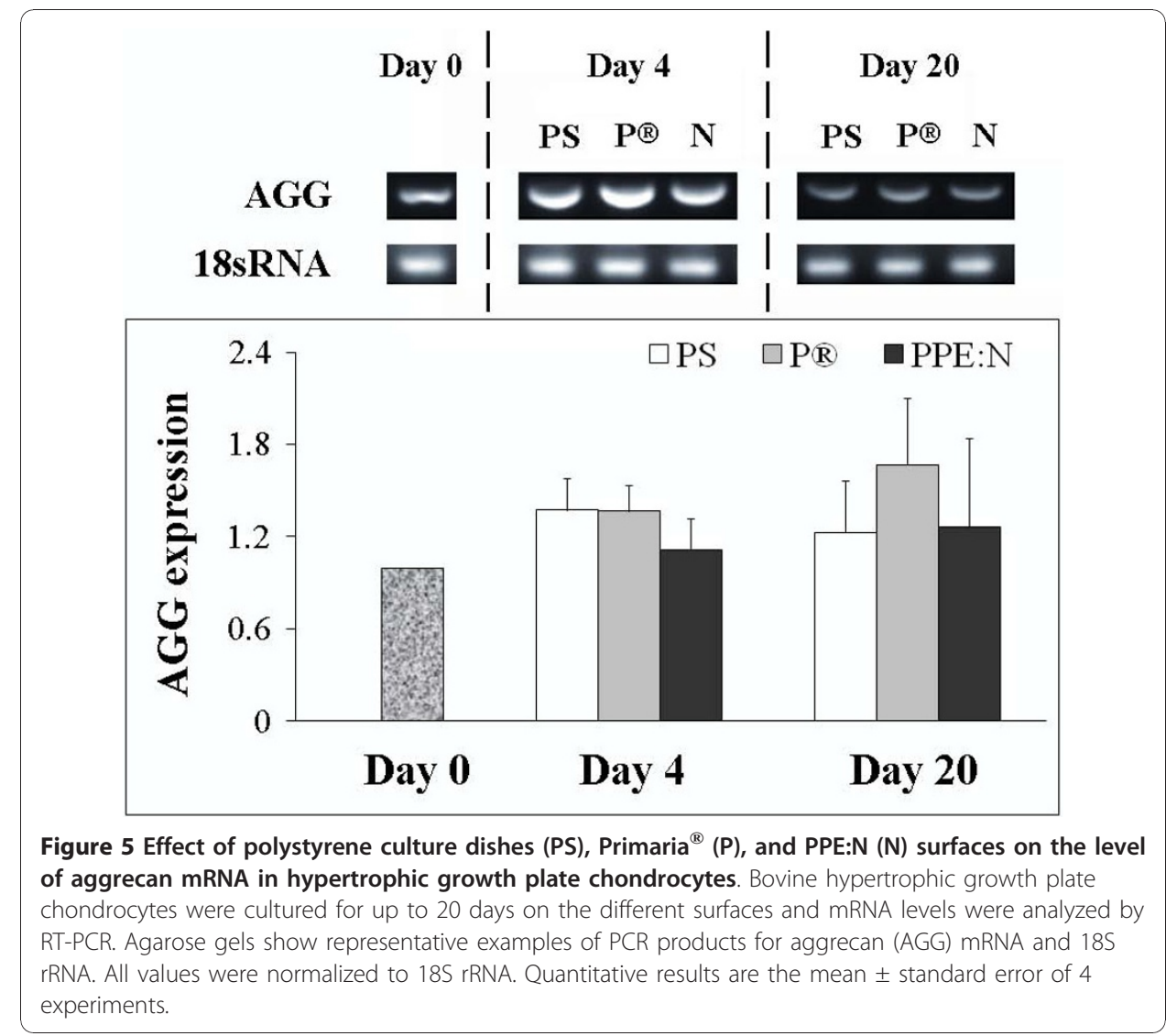

This, in turn, suggests that the effect of PPE:N may vary with the cell type. It is also possible that that the surfaces were altered by chondrocyte metabolism and lost their initial composition. This remains to be investigated. Nevertheless, we now know that the substrates' effect on adhering cells is mediated not by the absolute value of $[\mathrm{N}]$, but rather by the concentrations of various chemical functionalities at the surface, for example primary amines, imines, nitriles, amides $[18,29]$. However, hydroxyls (alcohols) and carboxylic acid groups can also play a role since bound oxygen is always incorporated in plasma polymer films due to the reaction of residual surface radicals with air. In the case of PS or Primaria ${ }^{\circledR}$, bound oxygen is due to plasma-modification of the polystyrene in an $\mathrm{O}_{2}$-containing gas mixture. As we recently reported elsewhere [18,29], we also know that primary amines account for 5 to $10 \%$ of $[\mathrm{N}]$. We also know that nitriles $(-\mathrm{C} \equiv \mathrm{N})$ also constitute an important surface functionality of PPE:N coatings. Moreover, primary amines are the dominant functionality in the remarkable adhesion behaviour we observed in the case of the U937 macrophages [29]. As a next step in the study of growth plate chondrocyte response on high-[N] culture surfaces, those earlier studies suggest working with coatings prepared by two other techniques in our laboratories, low-pressure plasma polymerisation [36], and vacuum ultraviolet photo-polymerisation [37], both capable of yielding a higher amount of primary amine than those found in the high-pressure plasma polymerised coatings used in this present work.

Finally, although gene expression data may suggest an influence of the nitrogen-rich surfaces on the hypertrophic phenotype, mineralization was not addressed in the present study. It is commonly believed that type X collagen is involved in controlling the 


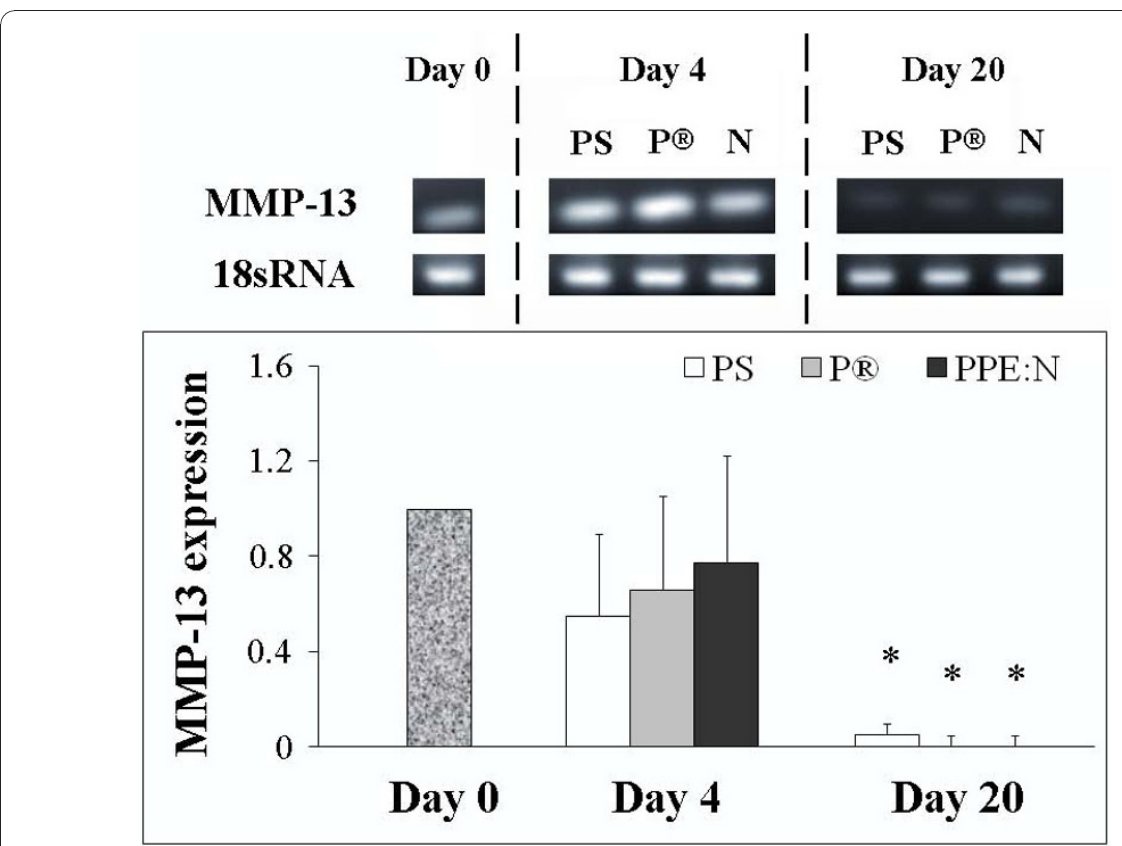

Figure 6 Effect of polystyrene culture dishes (PS), Primaria ${ }^{\circledR}(\mathrm{P})$, and PPE:N (N) surfaces on the level of MMP-13 mRNA in hypertrophic growth plate chondrocytes. Bovine hypertrophic growth plate chondrocytes were cultured for up to 20 days on the different surfaces and mRNA levels were analyzed by RT-PCR. Agarose gels show representative examples of PCR products for MMP-13 mRNA and 18S rRNA. All values were normalized to $18 \mathrm{~S}$ rRNA. Quantitative results are the mean \pm standard error of 4 experiments. * $p<0.05$ vs. day 4 .

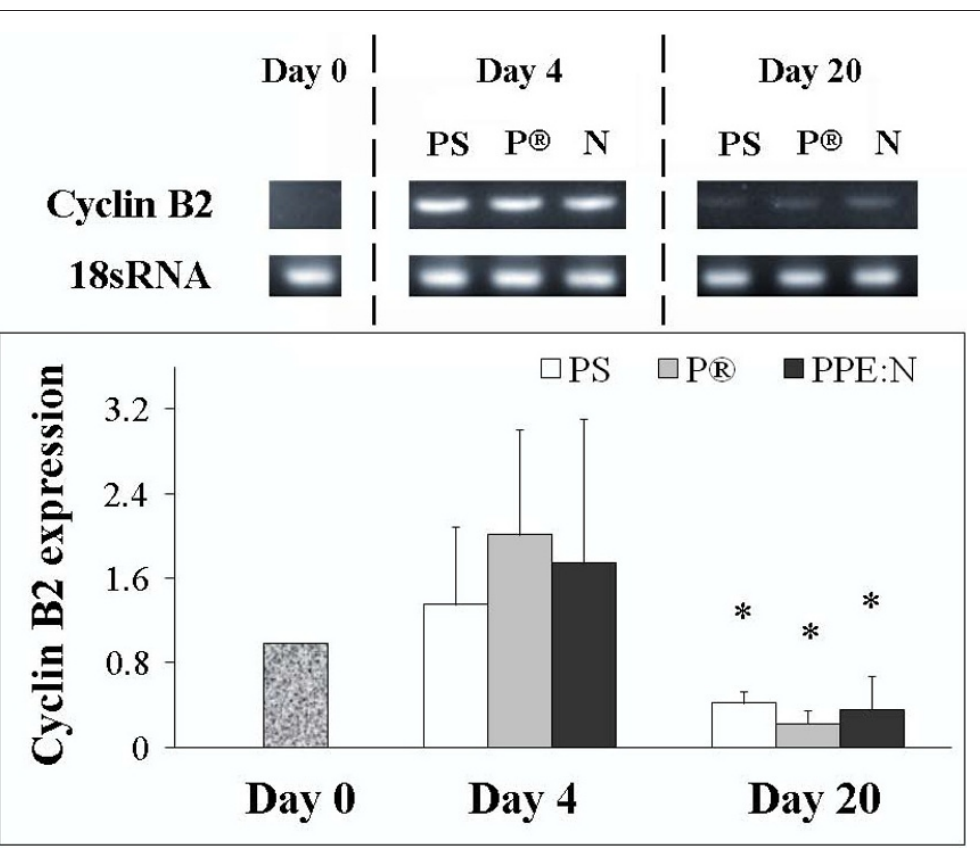

Figure 7 Effect of polystyrene culture dishes (PS), Primaria ${ }^{\circledR}(\mathrm{P})$, and PPE:N (N) surfaces on the level of cyclin B2 mRNA in hypertrophic growth plate chondrocytes. Bovine hypertrophic growth plate chondrocytes were cultured for up to 20 days on the different surfaces and mRNA levels were analyzed by RT-PCR. Agarose gels show representative examples of PCR products for cyclin B2 mRNA and 18S rRNA. All values were normalized to $18 \mathrm{~S} r R N A$. Quantitative results are the mean \pm standard error of 4 experiments. ${ }^{*} p<0.05$ vs. day 4 . 
later stages of endochondral bone formation [1-3]. In this case, culturing chondrocytes or MSCs on these surfaces should suppress mineralization. This is of great importance for cartilage repair or tissue engineering using MSCs from osteoarthritc patients known to express type X collagen. Further studies are therefore necessary to determine the effect of these surfaces on mineralization.

\section{Conclusions}

Type X collagen expression was reduced, at least transiently, by nitrogen rich surfaces in both growth plate chondrocytes as well as in MSCs from OA patients, which is beneficial to chondrogenesis. As "intelligent" surfaces, PPE:N coatings therefore represent a potentially advantageous cell-culture substrate with beneficial effects for cartilage and intervertebral disc repair. However, further studies are necessary to better understand the nature of specific functional groups, such as primary amines, on gene expression and cell phenotype: this information will be important in tissue engineering applications that require the use of such "intelligent" surfaces.

\section{Abbreviations}

BOPP: biaxially oriented polypropylene; $\mathrm{C}_{2} \mathrm{H}_{4}$ : ethylene; DMEM: Dulbecco's modified Eagle's medium; FBS: Fetal bovine serum; MMP: matrix metalloproteinase; MSC: Mesenchymal stem cell; N: Nitrogen; OA: Osteoarthritis; PPPE:N: N-doped plasma-polymerized ethylene; PS: Polystyrene; RT-PCR: Reverse transcriptase-Polymerase chain reaction; SCCM: standard cubic centimeters per minute; SLM: standard liters per minute; XPS: X-ray photoelectron spectroscopy

\section{Acknowledgements}

The authors gratefully acknowledge financial support from the Canadian Institutes of Health Research (CIHR), the AO Foundation (Switzerland), and the Natural Sciences and Engineering Research Council of Canada (NSERC).

\section{Author details}

${ }^{1}$ Lady Davis Institute for Medical Research, SMBD-Jewish General Hospital, 3755 Chemin de la Cote Ste-Catherine, Montreal, QC H3T 1E2, Canada. ²Division of Orthopaedic Surgery, McGill University Health Centre, 1650 Cedar Avenue, Montreal, QC H3G 1A4, Canada. ${ }^{3}$ Department of Engineering Physics, École Polytechnique, 2500, Chemin de Polytechnique, Montréal, QC H3T 1J4, Canada.

\section{Authors' contributions}

AP participated in the interpretation of the results, drafted the Introduction and the Discussion with FM, and revised the final version of the article. CND carried out experiments with stem cells, participated to RT-PCR experiments, and drafted the section on stem cell isolation and culture. PLGL prepared the surfaces and helped to draft the method section on surface preparation. DS participated to cell isolation and RT-PCR analysis. MRW critically revised the manuscript. JA participated in the design of the study and performed the statistical analysis. FW conceived the study and drafted the Introduction and the Discussion with AP. All authors read and approved the final manuscript.

\section{Competing interests}

The authors declare that they have no competing interests.

Received: 2 September 2010 Accepted: 18 January 2011 Published: 18 January 2011

\section{References}

1. Mwale F, Billinghurst C, Wu W, Alini M, Webber C, Reiner A, lonescu M, Poole J, Poole AR: Selective assembly and remodelling of collagens II and IX associated with expression of the chondrocyte hypertrophic phenotype. Dev Dyn 2000, 218:648-662.

2. Mwale F, Tchetina $E, W u$ CW, Poole AR: The assembly and remodeling of the extracellular matrix in the growth plate in relationship to mineral deposition and cellular hypertrophy: an in situ study of collagens II and IX and proteoglycan. J Bone Miner Res 2002, 17:275-283.

3. Tchetina E, Mwale F, Poole AR: Distinct phases of coordinated early and late gene expression in growth plate chondrocytes in relationship to cell proliferation, matrix assembly, remodeling, and cell differentiation. J Bone Miner Res 2003, 18:844-851.

4. Mwale F, Stachura D, Roughley P, Antoniou J: Limitations of using aggrecan and type X collagen as markers of chondrogenesis in mesenchymal stem cell differentiation. J Orthop Res 2006, 24:1791-1798.

5. Nelea V, Luo L, Demers CN, Antoniou J, Petit A, Lerouge S, Wertheimer MR, Mwale F: Selective inhibition of type X collagen expression in human mesenchymal stem cell differentiation on polymer substrates surface-modified by glow discharge plasma. J Biomed Mater Res Part A 2005, 75A:216-223.

6. Mwale F, Girard-Lauriault PL, Wang HT, Lerouge S, Antoniou J, Wertheimer MR: Suppression of hypertrophy and osteogenesis in committed human mesenchymal stem cells cultured on novel nitrogen-rich plasma polymer coatings. Tissue Eng 2006, 12:2639-2647. 
7. Mwale F, Wang HT, Nelea V, Luo L, Antoniou J, Wertheimer MR: The effect of glow discharge plasma surface modification of polymers on the osteogenic differentiation of committed human mesenchymal stem cells. Biomaterials 2006, 27:2258-2264.

8. Poole AR, Laverty S, Mwale F: Endochondral bone formation and development in the axial and appendicular skeleton. In The osteoporosis primer Edited by: Press CU 2000, 3-17.

9. Stanton LA, Underhill TM, Beier F: MAP kinases in chondrocyte differentiation. Dev Biol 2003, 263:165-715.

10. Wang G, Woods A, Sabari S, Pagnotta L, Stanton LA, Beier F: RhoA/ROCK signaling suppresses hypertrophic chondrocyte differentiation. J Biol Chem 2004, 279:13205-13214.

11. Stanton LA, Sabari S, Sampaio AV, Underhill TM, Beier F: p38 MAP kinase signalling is required for hypertrophic chondrocyte differentiation. Biochem J 2004, 378:53-62.

12. LuValle P, Beier F: Cell cycle control in growth plate chondrocytes. Front Biosci 2000, 5:D493-D503.

13. Beier $F$, Taylor $A C$, LuValle $P$ : Activating transcription factor 2 is necessary for maximal activity and serum induction of the cyclin A promoter in chondrocytes. J Biol Chem 2000, 275:12948-12953.

14. Beier F, Leask TA, Haque S, Chow C, Taylor AC, Lee RJ, Pestell RG, Ballock RT, LuValle P: Cell cycle genes in chondrocyte proliferation and differentiation. Matrix Biol 1999, 18:109-120.

15. Klein-Soyer C, Hemmendinger S, Cazenave J-P: Culture of human vascular endothelial cells on a positively charged polystyrene surface, Primaria: comparison with fibronectin-coated tissue culture grade polystyrene. Biomaterials 1989, 10:85-90.

16. Steele JG, Dalton BA, Johnson G, Underwood PA: Adsorption of fibronectin and vitronectin onto Primaria ${ }^{\circledR}$ and tissue culture polystyrene and relationship to the mechanism of initial attachment of human vein endothelial cells and BHK-21 fibroblasts. Biomaterials 1995, 16:1057-1067.

17. Girard-Lauriault P-L, Mwale F, lordanova M, Demers CN, Desjardins $P$, Wertheimer MR: Atmospheric pressure deposition of micropatterned N-rich plasma-polymer films for tissue engineering. Plasma Process Polym 2005, 2:263-270.

18. Girard-Lauriault PL, Desjardins P, Unger WES, Lippitz A, Wertheimer MR: Chemical characterisation of nitrogen-rich plasma-polymer films deposited in dielectric barrier discharges at atmospheric pressure. Plasma Process Polym 2008, 5:631-644.

19. Bullett NA, Bullett DP, Truica-Marasecu F, Lerouge S, Mwale F, Wertheimer MR: Polymer surface micropatterning by plasma and VUV-photochemical modification for controlled cell culture. Appl Surf Sci 2004, 235:395-405.

20. Guimond S, Radu I, Czeremuszkin G, Carlsson DJ, Wertheimer MR: Biaxially oriented polypropylene (BOPP) surface modification by nitrogen atmospheric pressure glow discharge (APGD) and by air corona. Plasmas Polym 2002, 7:71-88.

21. Pal S, Tang LH, Choi H, Habermann E, Rosenberg L, Roughley P, Poole AR: Structural changes during development in bovine fetal epiphyseal cartilage. Coll Relat Res 1981, 1:151-176.

22. Chomczynski P, Sacchi N: Single-step method of RNA isolation by acid guanidinium thiocyanate-phenol-chloroform extraction. Anal Biochem 1987, 162:156-159.

23. Wu CW, Tchetina EV, Mwale F, Hasty K, Pidoux I, Reiner A, Chen J, Van W, Poole AR: Proteolysis involving matrix metalloproteinase 13 (collagenase-3) is required for chondrocyte differentiation that is associated with matrix mineralization. J Bone Miner Res 2002, 17:639-651.

24. Acarturk TO, Peel MM, Petrosko P, LaFramboise W, Johnson PC, DiMilla PA: Control of attachment, morphology, and proliferation of skeletal myoblasts on silanized glass. J Biomed Mater Res 1999, 44:355-370.

25. Boyan $B D$, Hummert TW, Dean DD, Schwartz Z: Role of material surfaces in regulating bone and cartilage cell response. Biomaterials 1996, 17:137-146

26. Britland S, Clark P, Connolly P, Moores G: Micropatterned substratum adhesiveness: a model for morphogenetic cues controlling cell behavior. Exp Cell Res 1992, 198:124-129.

27. Hu Y, Winn SR, Krajbich I, Hollinger O: Porous polymer scaffolds surface-modified with arginine-glycine-aspartic acid enhance bone cell attachment and differentiation in vitro. J Biomed Mater Res Part A 2003, 64A:583-590.

28. Curran JM, Chen R, Hunt JA: Controlling the phenotype and function of mesenchymal stem cells in vitro by adhesion to silane-modified clean glass surfaces. Biomaterials 2005, 26:7057-7067.

29. Girard-Lauriault P-L, Truica-Marasescu F, Petit A, Wang HT, Desjardins P, Antoniou J, Mwale F, Wertheimer MR: Adhesion of human U937 monocytes to nitrogen-rich organic thin films: novel insights into the mechanism of cellular adhesion. Macromol Biosci 2009, 9:911-921.

30. Mwale F, Petit A, Wang HT, Epure LM, Girard-Lauriault P-L, Ouellet JA, Wertheimer MR, Antoniou J: The potential of Nrich plasma-polymerized ethylene (PPE:N) films for regulating the phenotype of the nucleus pulposus. The Open Orthop J 2008, 2:137-144.

31. Mwale F, Wang HT, Petit A, Girard-Lauriault P-L, Hunter CJ, Ouellet JA, Wertheimer MR, Antoniou J: The effect of novel nitrogen-rich plasma polymer coatings on the phenotypic profile of notochordal cells. Biomed Eng Online 2007, 6:33.

32. Salasznyk RM, Williams WA, Boskey A, Batorsky A, Plopper GE: Adhesion to vitronectin and collagen I promotes osteogenic differentiation of human mesenchymal stem cells. J Biomed Biotechnol 2004, 2004:24-34.

33. Li WJ, Tuli R, Huang X, Laquerriere $P$, Tuan RS: Multilineage differentiation of human mesenchymal stem cells in a three-dimensional nanofibrous scaffold. Biomaterials 2005, 26:5158-5166.

34. Mwale F, lordanova M, Demers CN, Steffen T, Roughley P, Antoniou J: Biological evaluation of chitosan salts crosslinked to genipin as a cell scaffold for disk tissue engineering. Tissue Eng 2005, 11:130-140.

35. Steinert A, Weber M, Dimmler A, Julius C, Schutze N, Noth U, Cramer H, Eulert J, Zimmermann U, Hendrich C: Chondrogenic differentiation of mesenchymal progenitor cells encapsulated in ultrahigh-viscosity alginate. J Orthop Res 2003, 21:1090-1097.

36. Truica-Marasescu F-E, Wertheimer MR: Nitrogen-rich plasma-polymer films for biomedical applications. Plasma Process Polym 2008, 5:44-57.

37. Truica-Marasescu F, Wertheimer MR: Vacuum ultraviolet (VUV) photo-polymerisation of amine-rich thin films. Macromol Chem Phys 2008, 209:1043-1049, \{Erratum: 2008, 209: 2061\}..

doi:10.1186/1475-925X-10-4

Cite this article as: Petit et al.: Effect of nitrogen-rich cell culture surfaces on type $\mathrm{X}$ collagen expression by bovine growth plate chondrocytes. BioMedical Engineering OnLine 2011 10:4. 